\title{
SIGNIFICANCE OF SERUM ALPHA-FETOPROTEIN LEVELS IN THE DIAGNOSIS AND PROGNOSIS OF PAEDIATRIC LIVER TUMOURS- A STUDY AT PAEDIATRIC REFERRAL CENTRE
}

\author{
O. H. Radhika Krishna ${ }^{1}$, K. Geetha², Sumaiah Fatima³, Srinivas Reddy P4, Ramesh Reddy Kota ${ }^{5}$ \\ ${ }_{1}^{1}$ Associate Professor, Department of Pathology, Osmania Medical College, Hyderabad, Telangana, India. \\ ${ }^{2}$ Assistant Professor, Department of Pathology, Niloufer Hospital, Telangana, India. \\ ${ }_{3}^{3}$ MBBS Student, Osmania Medical College, Hyderabad, Telangana, India. \\ ${ }^{4}$ Professor, Department of Paediatric Surgery, Niloufer Hospital, Telangana, India. \\ 5 Professor, Department of Paediatric Surgery, Niloufer Hospital, Telangana, India.
}

\section{ABSTRACT}

\section{BACKGROUND}

There is a need for accurate diagnosis and preoperative evaluation of paediatric liver tumours, because treatment modalities and management protocols vary for benign and malignant tumours. There is a widespread notion that elevated AFP levels are suggestive of malignant liver tumours. However, in paediatric age group, serum AFP levels are normally higher than adults upto eight months of age. Preoperative evaluation involves radiology and serum AFP levels.

So this study aims to study the AFP levels in both malignant and benign liver tumours and also understand if higher levels are seen in malignant liver tumours over their benign counterpart.

\section{MATERIALS AND METHODS}

A retrospective descriptive study of all the cases of paediatric liver tumours received at the Department of Pathology, Niloufer Hospital was done. Detailed clinical history, radiological investigations and serum AFP levels were recorded in all the cases.

\section{RESULTS}

The hypothesis that benign paediatric liver tumours show normal-to-mildly elevated AFP levels did not correlate with our results. There was a very wide variation in elevation of AFP levels in all the cases. Both benign and malignant liver tumours showed similar percentage of elevation in AFP levels.

\section{CONCLUSION}

AFP levels cannot be used as maker to predict malignancy in preoperative evaluation in paediatric age group due to significant elevation of AFP in both benign and malignant tumours.

\section{KEY WORDS}

Alpha-Fetoprotein, Hepatoblastoma, Paediatric Liver Tumours.

HOW TO CITE THIS ARTICLE: Krishna OHR, Geetha K, Fatima S, et al. Significance of serum alpha-fetoprotein levels in the diagnosis and prognosis of paediatric liver tumours- a study at paediatric referral centre. J. Evolution Med. Dent. Sci. 2018;7(41): 4457-4461, DOI: $10.14260 /$ jemds/2018/994

\section{BACKGROUND}

The liver is the site of third most common intra-abdominal malignancy following Wilms' tumour and neuroblastoma in the paediatric age group with an incidence of $0.4-1.9$ per million children per year.[1]

Malignant paediatric liver tumours include hepatoblastoma, angiosarcoma, rhabdomyosarcoma and constitute $70 \%$ of paediatric liver masses, whereas benign liver tumours like haemangiomas, haemangioendotheliomas and mesenchymal hamartomas account for $30 \%$ of all cases.[2]

'Financial or Other Competing Interest': None.

Submission 08-09-2018, Peer Review 25-09-2018,

Acceptance 28-09-2018, Published 08-10-2018.

Corresponding Author:

Dr. K. Geetha,

Assistant Professor,

Department of Pathology,

Niloufer Hospital,

Osmania Medical College,

Koti, Hyderabad, Telangana, India.

E-mail: geethakayalaasdfg@gmail.com

DOI: $10.14260 /$ jemds $/ 2018 / 994$ differentiation of liver masses into benign and malignant is still complex. Clinical findings, radiology, size and texture of tumour which are usually used to diagnose malignancy are very overlapping and thus pose problems to the clinician in arriving at a definite diagnosis.

There is a need for accurate diagnosis and preoperative evaluation of paediatric liver tumours, because treatment modalities and management protocols vary for benign and malignant tumours. Malignant tumours are subject to initial incisional biopsy for evaluation of the tumour followed by neoadjuvant chemotherapy. Unresectable malignant tumours resistant even to aggressive chemotherapy require complete hepatectomy and subsequent liver transplantation. Benign tumours, on the other hand, undergo a complete surgical resection as the mainstay of curative therapy.

Studies on paediatric liver tumours in India and South Asia are few. The correlation between AFP levels, clinical presentation and diagnosis has been little evaluated. The treating surgeon bases his approach on pre-surgical workup like clinical symptoms, radiological investigations, AFP and biopsy.

Any child suspected with liver tumour is tested for AFP levels due to the widespread notion that markedly elevated levels are suggestive of malignant tumours like hepatoblastoma. Furthermore, AFP levels are also used as a 
marker for response to treatment, prognosis and to determine the outcome of malignant liver tumours.[3] However, Dogan et al[4] reported that benign liver conditions like haemangioendotheliomas, mesenchymal hamartomas, cases of hepatic insult and regeneration like hepatitis and cirrhosis also show increased AFP levels. AFP monitoring is vital in management of hepatic tumours.(5) However, the significance of AFP levels as a diagnostic marker for malignant liver tumours is questionable and more so in the paediatric age group, where physiologically elevated levels in early postnatal life and upto 8 months of age make diagnosis based on AFP obscure.

The size of the liver mass, radiology or clinical history are of little utility in predicting malignancy. So, this study done at a paediatric tertiary referral hospital attempts to evaluate the incidence, variable clinical presentation and demographics of various paediatric liver tumours and correlate them with AFP levels as a predictor of malignancy. For this detailed clinical history, histopathological diagnosis and serum AFP levels are recorded and the utility of AFP levels as a predictor of malignancy is evaluated.

\section{Aims and Objectives}

To study the levels of serum AFP levels in malignant and benign liver tumours and also evaluate if the increase is more in malignant as compared to benign tumours of the liver.

\section{MATERIALS AND METHODS}

A retrospective descriptive study of all the cases of paediatric liver tumours received at the Department of Pathology, Niloufer Hospital, Hyderabad, for 2 years duration from June 2015 - June 2017 was done. The study population was 21 patients consisting of children below 12 years of age and the study period was for 2 months. Detailed clinical history, radiological investigations and serum AFP levels were recorded in all the cases.

\section{Inclusion Criteria}

All the paediatric patients with liver tumours, both benign and malignant on histopathology were included in this study.

\section{Exclusion Criteria}

Paediatric liver biopsy specimens for storage and cholestatic liver conditions were excluded from this study.

Serum AFP levels are measured using electrochemiluminescence immunoassay (ECLIA).

Liver function tests were done which include markers of liver function like bilirubin, Alanine aminotransferase (ALT or SGPT), Aspartate aminotransferase (AST or SGOT) and albumin.

Radiological investigations like ultrasonography and computed tomography as available were evaluated. Ultrasonography is useful to assess the tumour size, whether the mass is solid or cystic and the extent of the tumour within the liver. Abdominal and chest CT reveal the possibility of resectability and identify pulmonary metastasis.

For histopathological examination, open biopsies were obtained from the patients. A routine processing of the formalin fixed paraffin embedded tissue was done, stained by Haematoxylin and Eosin, studied for detailed histopathology and the final diagnosis was made.

\section{The Procedure is as follows}

\section{Fixation}

The specimen is immersed in $10 \%$ formalin solution for $6-12$ hours and placed in a labelled cassette for processing.

\section{Processing}

The specimen is then infiltrated with a sequence of dehydrating and clearing solvents like ethanol and xylene before they are placed in molten paraffin wax.

\section{Embedding}

The specimen is removed from the cassette and placed in wax filled mould, then the specimen block is allowed to solidify on a cold surface and when it is set the mould is removed.

\section{Sectioning}

Thin slices measuring 5 micrometres of the paraffin embedded specimen are made using a microtome for clear observation under the microscope.

\section{Staining}

A slice of the specimen is placed on the glass slide and stained with Haematoxylin and Eosin. Then it is observed under the microscope in 100x field.

The histological diagnosis was correlated with serum AFP levels and a significance was calculated and the role of AFP in diagnosis of paediatric liver tumours was evaluated.

For this, AFP levels of all the patients were classified into 4 groups:

Normal: $<20 \mathrm{ng} / \mathrm{mL}$, mildly elevated: $20-1000 \mathrm{ng} / \mathrm{dL}$, moderately elevated: 1000 - $10000 \mathrm{ng} / \mathrm{dL}$, remarkably elevated: $>10000 \mathrm{ng} / \mathrm{dL}$. Then the data was evaluated as to which type of liver tumour falls into which of the above categories of AFP levels. A correlation was done between age, tumour diagnosis and serum AFP levels

The statistical analysis could not be done due to the small sample size of the study.

\section{RESULTS}

21 histopathologically proven cases of liver tumours were taken up for the study, out of which 11 (52\%) cases were benign and $8(48 \%)$ were malignant. All the malignant cases were hepatoblastoma.

\begin{tabular}{|c|c|c|c|}
\hline $\begin{array}{c}\begin{array}{c}\text { Nature } \\
\text { of } \\
\text { Tumour }\end{array} \\
\end{array}$ & $\begin{array}{c}\text { Tumour } \\
\text { (HPE Diagnosis) }\end{array}$ & $\begin{array}{c}\text { No. } \\
\text { of } \\
\text { Cases }\end{array}$ & $\begin{array}{c}\text { \% Elevation } \\
\text { of AFP } \\
\text { Levels }\end{array}$ \\
\hline Malignant & Hepatoblastoma & 8 & $38 \%$ \\
\hline \multirow[b]{2}{*}{ Benign } & Mesenchymal hamartoma & 5 & $23.8 \%$ \\
\hline & $\begin{array}{c}\text { Infantile } \\
\text { Haemangioendothelioma }\end{array}$ & 2 & $9.5 \%$ \\
\hline \multirow[t]{2}{*}{$\mathrm{n}=11$} & Hydatid cyst & 4 & $19 \%$ \\
\hline & Simple hepatic cyst & 2 & $9.5 \%$ \\
\hline
\end{tabular}

Table 1. Number of Cases of Paediatric Liver Tumours 


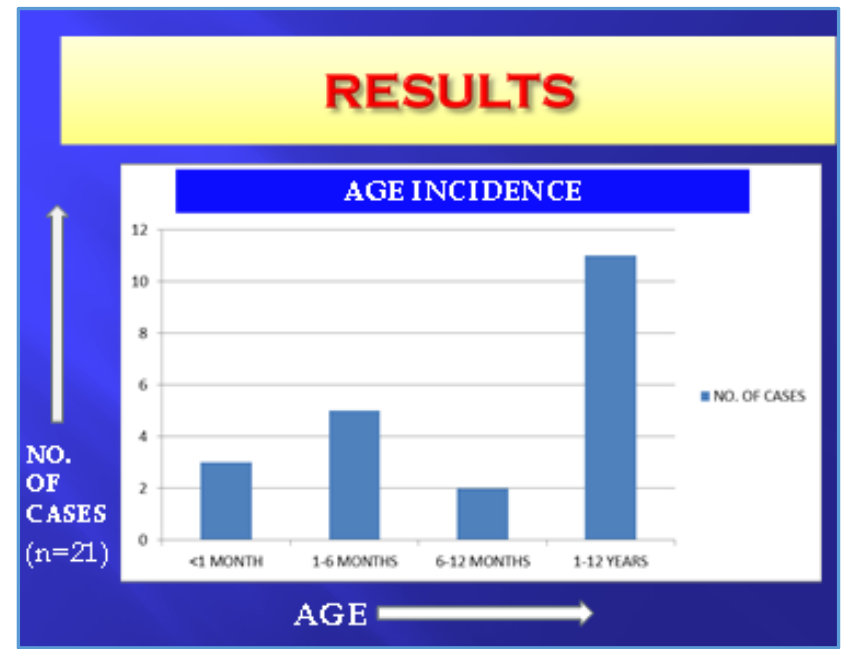

Most of the patients were older than one year of age. There were 7 male children and 14 female children. Liver function tests did not show any pattern. One case of hepatoblastoma had prolonged Activated Partial Thromboplastin Time (APTT), while one case of infantile haemangioendothelioma was detected with clinical jaundice and had bilirubin levels of $14.0 \mathrm{mg} / \mathrm{dL}$. LFT of one case of infantile haemangioendothelioma revealed increased alkaline phosphatase (ALP) levels.

\begin{tabular}{|c|c|c|c|c|}
\hline $\begin{array}{c}\text { Malignant } \\
\text { Tumour }\end{array}$ & Age & $\begin{array}{c}\text { AFP } \\
\text { Levels at } \\
\text { Diagnosis } \\
\text { (ng/mL) }\end{array}$ & \begin{tabular}{|c|} 
Normal \\
AFP Levels \\
according \\
to Age
\end{tabular} & $\begin{array}{c}\text { \% Elevation } \\
\text { of AFP } \\
\text { Levels }\end{array}$ \\
\hline \multirow{8}{*}{ 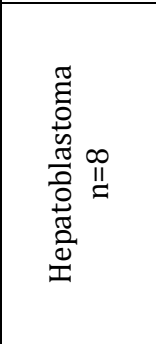 } & 20 days & 10,649 & $\begin{array}{c}9,452 \pm 12 \\
610\end{array}$ & Normal \\
\hline & 5 months & 3216 & $46.5 \pm 19$ & $6,816.12 \%$ \\
\hline & 1 year & 1999.6 & $4.7 \pm 3$ & $424.44 \%$ \\
\hline & $1^{1 / 2}$ years & 16,218 & $4.7 \pm 3$ & $3449.64 \%$ \\
\hline & $11 / 2$ years & 1210 & $4.7 \pm 3$ & $256.45 \%$ \\
\hline & 2 years & 1719 & $4.7 \pm 3$ & $364.74 \%$ \\
\hline & 2 years & 1428 & $4.7 \pm 3$ & 30 \\
\hline & 3 years & 4728 & $4.7 \pm 3$ & $1004.96 \%$ \\
\hline \multicolumn{5}{|c|}{$\begin{array}{c}\text { Table 2. Correlation of AFP Levels in Malignant Paediatric } \\
\text { Liver Tumour with Age }\end{array}$} \\
\hline
\end{tabular}

\begin{tabular}{|c|c|c|c|c|}
\hline Tumour & Age & \begin{tabular}{|c|} 
AFP \\
Levels at \\
Diagnosis \\
$(\mathrm{ng} / \mathrm{mL})$ \\
\end{tabular} & \begin{tabular}{|c|} 
Normal AFP \\
Levels \\
according to \\
Age
\end{tabular} & $\begin{array}{c}\% \\
\text { Elevation } \\
\text { of AFP } \\
\text { Levels } \\
\end{array}$ \\
\hline \multirow{5}{*}{$\begin{array}{c}\text { Mesenchymal } \\
\text { Hamartoma } \\
n=5\end{array}$} & 15 days & 57,823 & $\begin{array}{c}33,113 \pm \\
32503\end{array}$ & Normal \\
\hline & 15 days & 65,347 & $\begin{array}{c}33,113 \pm \\
32503\end{array}$ & Normal \\
\hline & 45 days & 638 & $323 \pm 278$ & Normal \\
\hline & 6 months & 38,132 & $12.5 \pm 9.8$ & $3049.56 \%$ \\
\hline & 11 months & 1417.9 & $4.7 \pm 3$ & $300.68 \%$ \\
\hline \multirow{2}{*}{$\begin{array}{c}\text { Infantile } \\
\text { Haemangio- } \\
\text { endothelioma } \\
n=2\end{array}$} & 45 & 180 & $2654 \pm 3080$ & Normal \\
\hline & 45 & 2154 & $2654 \pm 3080$ & Normal \\
\hline
\end{tabular}

9 cases were diagnosed as hepatoblastoma by Radiology, among which 8 were diagnosed as hepatoblastoma by histopathology and one turned out to be a haemangioendothelioma on histology.
All the cases diagnosed as haemangioendothelioma on Radiology were confirmed on histopathology.

Out of the total of 21 cases of paediatric liver tumours taken up for the study, AFP was found to be raised beyond age expected levels in 9 cases (42\%).

Out of these 9 cases, most of them 7 (77\%) were malignant hepatoblastoma and 2 (33\%) were benign mesenchymal hamartoma.

Out of the 8 malignant cases of histologically proven hepatoblastoma, only one case $(0.01 \%)$ showed normal serum AFP levels.

Out of the 11 benign liver tumours, $2(18 \%)$ showed increased serum AFP levels and remaining 9 (82\%) showed normal levels. In liver tumours occurring early in infancy, i.e. $<2$ months of age, AFP levels were found to correlate with the normal age matched values and did not raise significantly in both benign and malignant tumours.

All the cases of malignant Hepatoblastoma showed significant increase in AFP levels, except for the odd 20-dayold infant, in whom it was matching the age. Coming to benign, both the cases of haemangioendothelioma showed normal AFP levels; however, 2 among the 5 cases of mesenchymal hamartoma the commonest benign tumour in our study showed $300.68 \%$ and $3049.56 \%$ elevation in AFP levels for that particular age group. However, both the cases were 6 months or older, while the others were less than 2 months of age.

Considering the hypothesis that benign liver tumours show normal-to-mildly elevated AFP levels, there was a very wide variation in percentage elevation of AFP levels in all the cases irrespective of the diagnosis. Both benign and malignant tumours showed elevation in AFP levels.

\section{DISCUSSION}

9 cases were diagnosed as hepatoblastoma by Radiology, whereas 8 cases were diagnosed as hepatoblastoma by histopathology. This means that one benign liver tumour was mentioned as a possible malignancy by Radiology, suggesting that radiodiagnosis is not absolute in liver tumours.

Kassarjian A et al[ ${ }^{6]}$ reported that radiological findings do not always reflect the true liver pathology and in a study that initially identified 62 children with radiological diagnoses of infantile hepatic haemagioendotheliomas, 3 had malignant tumours based on tissue pathology. Radu Badea et al[7] reported that in arterialised haemangiomas and those with arteriovenous shunts, differentiation from a malignant tumour based on ultrasonography is difficult.

In liver tumours occurring early in infancy, i.e. $<2$ months of age, we found AFP levels were found to be within the normal reference range for that age. So measurement of serum AFP levels in children $<2$ months of age may not be useful in indicating the presence of liver tumours, because there might be an overlap between physiologically elevated levels in early infancy and increased AFP levels due to liver tumours in this age group.

AFP levels of hepatoblastoma followed by expected lines showed an elevation ranging from $256.45 \%$ in a $11 / 2$-year child to $6,816.12 \%$ in a 5-month-old child. However, the odd 20-day-old infant diagnosed as hepatoblastoma did not show raised AFP levels. Still raised AFP levels may have a predictive value in liver malignancy in paediatric age group. 
The following Ranges of AFP Levels in Hepatoblastoma have been reported in Literature-

AK Singal et al[8] reported in a study on tumour markers in paediatric solid tumours that AFP levels in hepatoblastoma can range from $<100 \mathrm{ng} / \mathrm{mL}$ to $>1,000,000 \mathrm{ng} / \mathrm{mL}$.

John A Sandoval et al[9] reported that most patients with hepatoblastoma have extremely high serum levels of AFP [upto $100,000 \pm 1,000,000$ times the normal value $(<10 \mathrm{ng} / \mathrm{mL})]$, but approximately $5 \%-10 \%$ of patients have unexpectedly low or even normal AFP values.

Van Tornout et al[3] reported in his study on 33 patients with confirmed hepatoblastoma that 4 patients had AFP levels in the range $100-999 \mathrm{ng} / \mathrm{mL}$ and 7 patients in the range of $10,000-99,999 \mathrm{ng} / \mathrm{mL}$ and 16 patients in the range of 100,000 - 999,999 $\mathrm{ng} / \mathrm{mL}$.

Contrary to the widespread notion that elevated AFP levels are suggestive of malignant liver tumours, in our study 2 cases of mesenchymal hamartoma showed $300.68 \%$ and $3049.56 \%$ elevation in AFP levels which are comparable to the \% elevation seen in hepatoblastoma. Both the children were older than 6 months of age. Only raised AFP level as a single marker may not be suggestive of malignancy.

In the literature, high AFP levels in benign liver tumours have been reported.

Sari et al[10] reported elevated AFP levels $(650 \mathrm{ng} / \mathrm{mL})$ in a 15-day-old boy with haemangioendothelioma (normal range for his age $88 \pm 87 \mathrm{ng} / \mathrm{mL}$ ). Another case of 11-monthold boy presenting with mesenchymal hamartoma had AFP levels of $3829 \mathrm{ng} / \mathrm{mL}$.

Dogan Kose et al[4] reported in the retrospective study done on 5 patients between 2006 and 2012 who were diagnosed with benign/ borderline liver masses that elevated AFP levels can be seen in benign liver masses and they can wrongly suggest malignant liver tumours. AFP levels were between $123 \mathrm{ng} / \mathrm{mL}$ and $4905 \mathrm{ng} / \mathrm{mL}$ in patients with median age of 6 months among whom 2 were diagnosed with haemangioendothelioma, 1 had mesenchymal hamartoma, 1 had haemangioma and 1 had focal nodular hyperplasia.

John D Murray et al[11] reported in the study of mesenchymal hamartoma in 8 patients that AFP was elevated in 1 patient $(108 \mathrm{ng} / \mathrm{mL})$. Liver function tests were normal in all patients.

Abraham H Dachman et al[12] reported in his study of infantile haemangioendothelioma of liver done on 27 patients that elevated AFP levels > $500 \mathrm{ng} / \mathrm{mL}$ were observed in 2 patients.

Although, the AFP levels were on the higher side in most of the hepatoblastomas, there still remained a case among the 8 cases of an infant aged 20 days in which it was normal. Similarly, AFP levels were normal in most benign tumours. There still were two cases out of 11 cases, both of mesenchymal hamartoma (18\%) that showed significant rise.

This study suggests that serum AFP level, when singly used as a diagnostic marker, is not helpful in pointing towards a specific diagnosis of benign or malignant. Raised levels do increase the suspicion of malignancy; however, a mesenchymal hamartoma the commonest and easily resectable tumour is still a possibility. Both the surgeon and the pathologist should be cautious about this, particularly in reporting tumour FNAC or small biopsies where histology might be overlapping.

\section{Limitation of the Study}

The statistical analysis could not be done due to small sample size.

\section{CONCLUSION}

Contrary to the widespread notion that elevated AFP levels are suggestive of liver malignancies. Serum AFP level alone may not be a reliable marker for the diagnosis of paediatric liver tumours.

Further study and research need to be done to find a diagnostic marker that can ascertain the nature of the tumour, whether benign or malignant, because treatment approach, management protocol and prognosis depends on the preoperative diagnosis of tumour.

Fractions of AFP like L3, P4 + P5 have been reported to allow differentiation of hepatocellular carcinoma from cirrhosis in some cases.[13] Another promising area of research is microRNAs, which are associated with hepatocellular carcinoma implicating their potential use in patient stratification of diagnosis and prognosis.[14]

There were no financial or personal relationships with other people or organisations that could inappropriately influence (bias) our work.

\section{REFERENCES}

[1] Reymond D, Plaschkes J, Luthy AR, et al. Focal nodular hyperplasia of the liver in children: review of followup and outcome. J Pediatr Surg 1995;30(11):1590-3.

[2] Reynolds M. Pediatric liver tumors. Semin Surg Oncol 1999;16(2):159-72.

[3] Van Tornout JM, Buckley JD, Quinn JJ, et al. Timing and magnitude of decline in alpha-fetoprotein levels in treated children with unresectable or metastatic hepatoblastomas are predictors of outcome: a report from the Children's Cancer Group. Journal of Clinical Oncology 1997;15(3):1190-7.

[4] Kose D, Erol C, Koksal Y. Elevated alpha-fetoprotein in benign/borderline liver masses in children. Turk Onkoloji Dergisi 2015;30(1):1-4.

[5] Yadav SP, Dhingra N. Treating pediatric liver tumors in India: a challenging proposition. Indian Pediatr 2012;49:839.

[6] Kassarjian A, Zurakowski D, Dubois J, et al. Infantile hepatic hemangiomas: clinicaland imaging findings and their correlation with therapy. AJR Am J Roentgenol 2004;182(3):785-95.

[7] Badea R, Ioanitescu S. Ultrasound imaging if liver tumors-current clinical applications. In: Alexander J, edr. Liver tumors. 2012: p. 75-103.

[8] Singal AK, Agarwala S. Tumour markers in pediatric solid tumours. J Indian Assoc Pediatr Surg 2005;10(3):183-90.

[9] Sandoval JA, Malkas LH, Hickey RJ. Clinical significance of serum biomarkers in pediatric solid mediastinal and abdominal tumors. Int J Mol Sci 2012;13(1):1126-53.

[10] Sari N, Yalçin B, Akyüz C, et al. Infantile hepatic hemangioendothelioma with elevated serum alphafetoprotein. Pediatr Hematol Oncol 2006;23(8):63947. 
[11] Murray JD, Ricketts RR. Mesenchymal hamartoma of the liver. The American Surgeon 1998;64(11):1097103.

[12] Dachman AH, Lichtenstein JE, Friedman AC, et al. infantile hemangioendothelioma of the liver: a radiologic-pathologic-clinical correlation. AJR 1983;140:1091-6.
[13] Stocker JT. Hepatoblastoma. Semin Diagn Pathol 1994;11(2):136-43.

[14] Dehner LP, Ishak KG. Vascular tumors of the liver in infants and children. A study of 30 cases and review of the literature. Arch Pathol 1971;92(2):101-11. 